\title{
Einstein-Dirac theory on gauge-natural bundles
}

\author{
Paolo Matteucci*
}

$14^{\text {th }}$ September 2003

\begin{abstract}
We present a clear-cut example of the importance of the functorial approach of gaugenatural bundles and the general theory of Lie derivatives for classical field theory, where the sole correct geometrical formulation of Einstein (-Cartan) gravity coupled with Dirac fields gives rise to an unexpected indeterminacy in the concept of conserved quantities.

Math. Subj. Class. (2000): primary: 83C40; secondary: 53C80, 83D05

Keywords: Einstein-Cartan-Dirac theory; conserved quantities; gauge-natural bundles; Lie derivative of spinor fields
\end{abstract}

\section{Introduction}

It is commonly accepted nowadays that the appropriate mathematical arena for classical field theory is that of fibre bundles or, more precisely, of their jet prolongations $[1,45,39,19]$. What is less often realized or stressed is that, in physics, fibre bundles are always considered together with some special class of morphisms, i.e. as elements of a particular category. The category of natural bundles was introduced about thirty years ago $[33,38,43,28]$ and proved to be an extremely fruitful concept in differential geometry $[35,9,25,8,32]$. But it was not until recently, when a suitable generalization was introduced, that of gauge-natural bundles $[7,28]$, that the relevance of this functorial approach to physical applications began to be clearly perceived [12, 21, 10, 13, 22, 15, 14].

Indeed, every classical field theory can be regarded as taking place on some jet prolongation of some gauge-natural (vector or affine) bundle associated with some principal bundle over a given base manifold $[7,28,10]$.

On the other hand, it is well known that one of the most powerful tools of Lagrangian field theory is the so-called "Noether theorem" $[34,28,19]$. It turns out that, when phrased in modern geometrical terms, this theorem crucially involves the concept of a Lie derivative, and here is where the aforementioned functorial approach is not only useful, but also intrinsically unavoidable. On relying on the general theory of Lie derivatives $[44,24,28,11,20]$, it is easy to see that the concept of Lie differentiation is, crucially, a category-dependent one, and it makes a real difference in taking the Lie derivative of, say, a vector field if one regards the tangent bundle as a purely natural bundle or, alternatively, as a more general gauge-natural bundle associated with some suitable principal bundle ( $c f .[20])$.

In this paper, we show that this functorial approach is essential for a correct geometrical formulation of the Einstein (-Cartan) -Dirac theory and, at the same time, yields an unexpected indeterminacy in the concept of conserved quantities. In the Einstein-Cartan-Dirac case, such an indeterminacy can be regarded as the well-known indeterminacy which occurs in gauge theory $[34,19,10,2]$, although there are serious conceptual risks involved in dismissing this "metricaffine" theory of gravitation as a standard "gauge theory" [45, 19]. This is certainly not the case, though, for the Einstein-Dirac theory proper, which can by no means be viewed as such. We shall

*Faculty of Mathematical Studies, University of Southampton, Highfield, Southampton SO17 1BJ, England (UK). E-mail address: p.matteucci@maths.soton.ac.uk. 
show that, in both cases, this indeterminacy actually arises from the very fact that, when coupled with Dirac fields, Einstein's general relativity can no longer be regarded as a purely natural theory because, in order to incorporate spinors, one must enlarge the class of morphisms of the theory.

Indeed, it is well-known that there are no representations of the group $\operatorname{GL}(4, \mathbb{R})$ of the automorphisms of $\mathbb{R}^{4}$ which behave like spinors under the subgroup of Lorentz transformations. Therefore, if one aims at considering the coupling between general relativity and fermionic fields, one is forced to resort to the so-called "tetrad formalism" (cf., e.g., [47]). Yet, there seems to have been a widespread misunderstanding of the full mathematical (and physical) significance of this. Leaving all the technicalities to the later sections, it will suffice here to sketchily recall how the concept of a tetrad is usually introduced.

On relying on the "principle of equivalence", which mathematically is tantamount to the simple statement that every manifold is locally flat, at every point $\tilde{x}$ of space-time one can erect a set of coordinates $\left(X^{a}\right)$ that are locally inertial at $\tilde{x}$. The components of the metric in any general non-inertial coordinate system are then ${ }^{1}$

$$
g_{\mu \nu}(x)=\theta^{a}{ }_{\mu}(x) \theta^{b}{ }_{\nu}(x) \eta_{a b},
$$

where $\left\|\eta_{a b}\right\|:=\operatorname{diag}(1,-1,-1,-1)$ and

$$
\theta^{a}{ }_{\mu}(\tilde{x}):=\left.\frac{\partial X^{a}(x)}{\partial x^{\mu}}\right|_{x=\tilde{x}},
$$

Thus, if we change our general non-inertial coordinates from $\left(x^{\mu}\right)$ to $\left(x^{\prime \mu}\right), \theta^{a}{ }_{\mu}$ will change according to the rule

$$
\theta^{a}{ }_{\mu} \mapsto{\theta^{\prime a}}_{\mu}=\frac{\partial x^{\nu}}{\partial x^{\prime \mu}} \theta^{a}{ }_{\nu} .
$$

Therefore, $\left(\theta^{a}{ }_{\mu}\right)$ must be regarded as the components of four 1-forms $\left(\theta^{a}\right)$, not of a single tensor field $\theta$. This set of four 1 -forms is what is known as a tetrad.

At this stage, the Latin index $a$ is just a "label" and, for any $a, \theta^{a}$ is indeed a natural object, i.e., roughly speaking, a section of a fibre bundle over the space-time manifold $M$ such that every coordinate change on the fibre is induced by some coordinate change on $M$. But the reason why a tetrad was introduced in the first place is precisely that we then wanted to "switch on" that Latin index in order to incorporate spinors into our formalism. This means that $\theta^{a}{ }_{\mu}$ will have to additionally change according to the rule

$$
\theta^{a}{ }_{\mu}(x) \mapsto L_{b}^{a}(x) \theta^{b}{ }_{\mu}(x),
$$

where $L(x)$ is the (space-time-dependent) Lorentz transformation induced (modulo a sign) by a given spinorial transformation $S$ under the group epimorphism $\Lambda: \operatorname{Spin}(1,3)^{e} \rightarrow \mathrm{SO}(1,3)^{e}$.

This is precisely the point that has been too often overlooked. Unlike (iii), transformation law $(i v)$ does not descend from definition (ii), but is a requirement we have imposed a posteriori. In other words, we have changed the definition of $\theta^{a}{ }_{\mu}$ in such a way that now $\left(\theta^{a}{ }_{\mu}\right)$ must be regarded as the components of a non-natural object $\theta$.

There is another important point that has been traditionally overlooked, which is of preeminent physical significance. Recall, indeed, that spinor fields can be defined on a manifold $M$ only if $M$ admits a "spin structure". Now, the standard definition of a spin structure involves fixing a metric on $M$, a framework which is certainly well-suited to a situation in which the gravitational field is considered unaffected by spinors, but is otherwise unable to describe the complete interaction and feedback between gravity and spinor fields [46, 41]. To this end, the concept of a free spin structure must be introduced.

Ultimately, the solution to both the aforementioned problems lies in suitably defining the bundle of which $\theta$ is to be a section. This leads to the concept of a spin-tetrad, which turns out to be a gauge-natural object $[12,21,22]$.

\footnotetext{
${ }^{1}$ Here and in the sequel both Latin and Greek indices range from 0 to 3.
} 
In the hope of making the paper understandable to both physicists and mathematicians, we have tried to make it as self-contained as possible. Its structure is the following: in $\S 1$ preliminary notions on jets, principal bundle morphisms and Clifford algebras are recalled for the main purpose of fixing our notation. In $\S 2$ gauge-natural bundles and a generalized notion of a Lie derivative are introduced. In $\S 3$ a version of Noether's theorem suitable for gauge-natural bundles is given. In $\S 4$ the concepts of a spin-tetrad and a spin-connection are defined. Finally, in $\S 5$ we briefly recall the Lagrangian formulation of the Einstein (-Cartan) -Dirac theory and, on applying the theory of conserved quantities, find a general superpotential, which essentially proves the aforementioned indeterminacy of any conserved charge associated with the gravitational field.

\section{Preliminaries and notation}

Throughout the paper, we shall assume that all maps are smooth, i.e. of class $C^{\infty}$, and all manifolds are real, finite-dimensional, Hausdorff, second-countable and, hence, paracompact.

\subsection{Jets}

We assume that the reader is familiar with the standard concepts and language of differential geometry on fibred manifolds, jet prolongation theory and calculus of variations on fibred manifolds. Standard references are [39, 28, 19].

Let $\pi: B \rightarrow M$ be a fibred manifold. We shall denote by $\Gamma(B)$ the space of all its (local) sections and set $m:=\operatorname{dim} M$ and $n:=\operatorname{dim} B-m$. On $B$ we shall use fibred charts $\left(V, x^{\lambda}, y^{\mathfrak{a}}\right)$, $\lambda=0, \ldots, m-1, \mathfrak{a}=1, \ldots, n$, where $V$ is an open subset of $B$ projecting on the domain $U$ of a chart $\left(U, x^{\lambda}\right)$ of $M$. If $\pi^{\prime}: M^{\prime} \rightarrow B^{\prime}$ is another fibred manifold, by a fibred (manifold) morphism between $B$ and $B^{\prime}$ we shall mean a pair $(\varphi, \Phi)$, where $\varphi \in C^{\infty}\left(M, M^{\prime}\right), \Phi \in C^{\infty}\left(B, B^{\prime}\right)$ and $\pi^{\prime} \circ \Phi=\varphi \circ \pi$. In particular, a base-preserving (fibred) morphism from $B$ to $B^{\prime}$ will be a fibred morphism between $B$ and $B^{\prime}$ for which $M^{\prime} \equiv M$ and $\varphi \equiv \operatorname{id}_{M}$.

Recall that two curves $\gamma, \delta: \mathbb{R} \rightarrow M$ are said to have contact of order $k$ at zero if, for every smooth function $\varphi: M \rightarrow \mathbb{R}$, all derivatives up to order $k$ of the difference $\varphi \circ \gamma-\varphi \circ \delta$ vanish at $0 \in \mathbb{R}$. Two maps $f, g: M \rightarrow N$ are then said to determine the same $k$-jet at $x \in M$ if, for every curve $\gamma: \mathbb{R} \rightarrow M$ with $\gamma(0)=x$, the curves $f \circ \gamma$ and $g \circ \gamma$ have contact of order $k$ at zero, and we shall write $j_{x}^{k} f=j_{x}^{k} g$. In particular, let $\pi: B \rightarrow M$ be a fibred manifold: the set $J^{k} B$ of all $k$-jets of its local sections has a natural topology of a fibred manifold over $M$, denoted by $\pi^{k}: J^{k} B \rightarrow M$ and called the $k$-th order jet prolongation of $\pi: B \rightarrow M$. If $\pi: B \rightarrow M$ is a bundle, so is $\pi^{k}: J^{k} B \rightarrow M$. Its holonomic sections are called $k$-th order jet prolongations of sections of $\pi: B \rightarrow M$ and will be denoted by $j^{k} \sigma$ for any given $\sigma \in \Gamma(B)$. The adapted fibred chart on $J^{k} B$ induced by the chart $\left(V, x^{\lambda}, y^{\mathfrak{a}}\right)$ on $B$ will be denoted by $\left(J^{k} V, x^{\lambda}, y^{\mathfrak{a}} \boldsymbol{\mu}\right)$, where $\boldsymbol{\mu}$ is a multi-index of length $|\boldsymbol{\mu}|$ such that $0 \leqslant|\boldsymbol{\mu}| \leqslant k$. Moreover, we shall set $\partial_{\lambda}:=\partial / \partial x^{\lambda}, \partial_{\mathfrak{a}}{ }^{\mu}:=\partial / \partial y^{\mathfrak{a}}{ }_{\boldsymbol{\mu}}$ and $J^{0} B:=B$, as customary.

Let $\mathcal{F}(B)$ denote the ring of smooth, real-valued functions over $B$. We define an $\mathcal{F}(B)$ submodule $\Omega_{0}^{p}(B)$ of the module $\Omega^{p}(B)$ of $p$-forms over $B$ according to the following prescription: $\omega \in \Omega_{0}^{p}(B)$ iff $\left.\Upsilon\right\lrcorner \omega=0$ for any vertical vector field $\Upsilon$ on $B$ (i.e. any vector field $\Upsilon$ such that $T \pi \circ \Upsilon=0)$, ' $\lrcorner$ ' denoting the interior product. The elements of $\Omega_{0}^{p}(B)$ are called horizontal p-forms (on $B$ ) and are in one-to-one correspondence with the base-preserving morphisms from $B$ to $\bigwedge^{p} T^{*} M$. Furthermore, we say that $\omega \in \Omega^{p}\left(J^{k} B\right)$ is a contact $p$-form iff $\left(j^{k} \sigma\right)^{*} \omega=0$ for all local section $\sigma$ of $B$.

Now, let $\pi_{h}^{k}: J^{k} B \rightarrow J^{h} B, k \geqslant h$, be the canonical projection. Recall that for any $p$-form $\omega \in \Omega^{p}\left(J^{k} B\right), p \leqslant m$, there exists a unique invariant decomposition

$$
\left(\pi_{k}^{k+1}\right)^{*} \omega=h(\omega)+k(\omega)
$$

into its horizontal part $h(\omega) \in \Omega_{0}^{p}\left(J^{k+1} B\right)$ and contact part $k(\omega) \in \Omega^{p}\left(J^{k+1} B\right)$. In particular, given a function $f \in \mathcal{F}\left(J^{k} B\right)$, in any fibred chart we have $h(\mathrm{~d} f):=\mathrm{d}_{\mu} f \mathrm{~d} x^{\mu}$, where $\mathrm{d}_{\mu} f$ denotes 
the formal or total (coordinate) derivative of $f$, defined by requiring

$$
\left(\mathrm{d}_{\mu} f\right) \circ j^{k+1} \sigma=\partial_{\mu}\left(f \circ j^{k} \sigma\right)
$$

for all $\sigma \in \Gamma(B)$. We shall also write $\mathrm{d}_{\boldsymbol{\mu}}, 1 \leqslant|\boldsymbol{\mu}| \leqslant k$, for $\mathrm{d}_{\mu_{k}} \cdots \mathrm{d}_{\mu_{1}}$. Analogously, we define the horizontal differential $\mathrm{d}_{\mathrm{H}} \omega$ of any form $\omega \in \Omega^{p}\left(J^{k} B\right)$ by requiring

$$
\left(j^{k+1} \sigma\right)^{*} \mathrm{~d}_{\mathrm{H}} \omega=\left(j^{k} \sigma\right)^{*} \mathrm{~d} \omega \equiv \mathrm{d}\left[\left(j^{k} \sigma\right)^{*} \omega\right]
$$

for all $\sigma \in \Gamma(B)$.

\subsection{Principal bundle morphisms}

For the reader's convenience, we recall herein some basic ideas on principal bundle morphisms ( $c f$. , e.g., [27]).

Let $P(M, G)$ be a principal (fibre) bundle. A (principal) automorphism of $P$ is a $G$-equivariant diffeomorphism of $P$ onto itself, i.e. a diffeomorphism $\Phi: P \rightarrow P$ such that $\Phi(u \cdot a)=\Phi(u) \cdot a$ for all $u \in P$ and $a \in G$, '.' denoting the canonical right action of $G$ on $P$. We shall denote by $\operatorname{Aut}(P)$ the group of all automorphisms of $P$.

Now, let $\Xi$ be a vector field on $P$ generating a one-parameter group $\left\{\Phi_{t}\right\}$. Then, $\Xi$ is called $G$-invariant if $\Phi_{t}$ is an automorphism of $P$ for all $t \in \mathbb{R}$.

Owing to $G$-equivariance, each automorphism $\Phi \in \operatorname{Aut}(P)$ induces a unique diffeomorphism $\varphi: M \rightarrow M$ such that $\pi \circ \Phi=\varphi \circ \pi, \pi$ denoting the canonical projection of $P$ on $M$. Then it follows immediately that every $G$-invariant vector field $\Xi$ on $P$ is projectable over a unique vector field $\xi$ on the base manifold $M$, i.e. $T \pi \circ \Xi=\xi \circ \pi$.

\subsection{Clifford algebra, $\gamma$ matrices and spin group}

The Clifford algebra $\mathcal{C} \ell(V)$ on a (real) vector space $V$ equipped with a scalar product $(u, v) \mapsto$ $g(u, v)$ is an associative algebra such that there exists a linear map $\gamma$ from $V$ into a subset of $\mathcal{C} \ell(V)$ generating $\mathcal{C} \ell(V)$ and satisfying the property

$$
\gamma(u) \gamma(v)+\gamma(v) \gamma(u)=-2 g(u, v) e,
$$

$e$ denoting the unit element of $\mathcal{C} \ell(V)$. The Clifford algebra on an $m$-dimensional vector space has dimension $2^{m}$. It can be realized by an algebra of linear maps of a complex vector space of dimension $2^{I(m / 2)}$ into itself, $I(m / 2)$ denoting the integral part of $m / 2$.

By $\gamma$ matrices we shall mean a set of $m$ such linear maps, represented by matrices, associated with the vectors of an orthonormal frame of $V$.

If we denote by $\left(\eta_{a b}\right)$ the components of $g$ in such a frame, then the $\gamma$ matrices, which we shall denote by $\left(\gamma_{a}\right)$, satisfy the fundamental relation

$$
\gamma_{a} \gamma_{b}+\gamma_{b} \gamma_{a}=-2 \eta_{a b}
$$

where the identity matrix is implied on the right-hand side. We shall also define

$$
\gamma_{a_{1} \ldots a_{k}}:=\frac{1}{k !} \gamma_{\left[a_{1}\right.} \cdots \gamma_{\left.a_{k}\right]} .
$$

In fact, it turns out that we need to consider only antisymmetrized products. This is because, on applying (1.1) iteratively, we find

$$
\begin{gathered}
\gamma_{a} \gamma_{b}=\gamma_{a b}-\eta_{a b} \\
\gamma_{a} \gamma_{b} \gamma_{c}=\gamma_{a b c}-\eta_{a b} \gamma_{c}-\eta_{b c} \gamma_{a}+\eta_{c a} \gamma_{b}
\end{gathered}
$$

and so forth. Moreover, substituting the former into the latter relation yields

$$
\gamma_{a b} \gamma_{c}+\gamma_{c} \gamma_{a b}=2 \gamma_{a b c},
$$


an identity which will prove useful later on.

Henceforth, $V$ will be assumed to be 4 -dimensional and $g$ will have signature $(1,3)$. Therefore, $\left\|\eta_{a b}\right\|=\operatorname{diag}(1,-1,-1,-1)$. Furthermore, it can be shown that the $\gamma$ matrices satisfy the following [anti] Hermiticity properties:

$$
\gamma_{a}^{\dagger}= \begin{cases}-\gamma_{a} & \text { if } a=0, \\ \gamma_{a} & \text { if } a=1,2,3,\end{cases}
$$

' $\dagger$ ' denoting transposition and complex conjugation. From (1.1) and (1.3) it follows immediately that

$$
\gamma_{0} \gamma_{a} \gamma_{0}^{-1}=-\gamma_{a}^{\dagger}
$$

Finally, by the spin group $\operatorname{Spin}(1,3)$ we shall mean the subgroup of $\mathrm{GL}(4, \mathbb{C})$ consisting of those elements $S$ such that there exists an $L \in \mathrm{SO}(1,3)$ satisfying

$$
S \gamma_{a} S^{-1}=L_{a}{ }^{b} \gamma_{b}
$$

$L \equiv\left\|L_{a}^{b}\right\|$, and such that

$$
\operatorname{det}(S)=1
$$

Relations (1.4) define an epimorphism from $\operatorname{Spin}(1,3)$ onto $\mathrm{SO}(1,3)$. It can be shown that $\operatorname{Spin}(1,3)\left[\operatorname{Spin}(1,3)^{e}\right]$ is the twofold covering of $\mathrm{SO}(1,3)\left[\mathrm{SO}(1,3)^{e}\right]$, the superscript $e$ denoting the connected component with the unit. In particular, $\operatorname{Spin}(1,3)^{e}$ is simply connected.

\section{Gauge-natural bundles}

The concept of a gauge-natural bundle was originally introduced by Eck in [7]. As we have applications in mind, in this section we shall follow a constructive approach along the lines of [28], notably $\S 15$ and $\S 52.4$. An (equivalent) axiomatic formulation can be found again in [28, Chapter XII].

Definition 2.1. The set

$$
\left\{j_{0}^{k} \alpha \mid \alpha: \mathbb{R}^{m} \rightarrow \mathbb{R}^{m}, \alpha(0)=0, \text { locally invertible }\right\}
$$

equipped with the jet composition $j_{0}^{k} \alpha \circ j_{0}^{k} \alpha^{\prime}:=j_{0}^{k}\left(\alpha \circ \alpha^{\prime}\right)$ is a Lie group called the $k$-th differential group and denoted by $G_{m}^{k}$.

For $k=1$ we have, of course, the identification $G_{m}^{1} \cong \mathrm{GL}(m, \mathbb{R})$.

Definition 2.2. Let $M$ be an $m$-dimensional manifold. The principal bundle over $M$ with group $G_{m}^{k}$ is called the $k$-th order frame bundle over $M$ and will be denoted by $L^{k} M$.

For $k=1$ we have, of course, the identification $L^{1} M \cong L M$, where $L M$ is the usual (principal) bundle of linear frames over $M$ (cf., e.g., [27]).

Definition 2.3. Let $G$ be a Lie group. Then, by the space of $(m, h)$-velocities of $G$ we shall mean the set

$$
T_{m}^{h} G:=\left\{j_{0}^{h} a \mid a: \mathbb{R}^{m} \rightarrow G\right\} .
$$

Thus, $T_{m}^{h} G$ denotes the set of $h$-jets with "source" at the origin $0 \in \mathbb{R}^{m}$ and "target" in $G$, and can be given the structure of a (Lie) group. Indeed, let $S, T \in T_{n}^{h} G$ be any elements. We define a (smooth) multiplication in $T_{m}^{h} G$ by:

$$
T_{m}^{h} \mu: T_{m}^{h} G \times T_{m}^{h} G \rightarrow T_{m}^{h} G, \quad\left(S=j_{0}^{h} a, T=j_{0}^{h} b\right) \mapsto S \cdot T:=j_{0}^{h}(a \cdot b),
$$

where $(a \cdot b)(x):=a(x) \cdot b(x) \equiv \mu(a(x), b(x))$ is the group multiplication in $G$. The mapping $(S, T) \mapsto S \cdot T$ is associative; moreover, the element $j_{0}^{h} e, e$ denoting both the unit element in $G$ and the constant mapping from $\mathbb{R}^{n}$ onto $e$, is the unit element of $T_{n}^{h} G$, and $j_{0}^{h} a^{-1}$, where $a^{-1}(x):=$ $(a(x))^{-1}$ (the inversion being taken in the group $G$ ), is the inverse of $j_{0}^{h} a$. 
Definition 2.4. Consider a principal bundle $P(M, G)$. Let $k$ and $h$ be two natural numbers such that $k \geqslant h$. Then, by the $(k, h)$-principal prolongation of $P$ we shall mean the bundle

$$
W^{k, h} P:=L^{k} M \times_{M} J^{h} P,
$$

$J^{h} P$ denoting the $h$-th order jet prolongation of $P$. A point of $W^{k, h} P$ is of the form $\left(j_{0}^{k} \epsilon, j_{x}^{h} \sigma\right)$, where $\epsilon: \mathbb{R}^{m} \rightarrow M$ is locally invertible with $\epsilon(0)=x$, and $\sigma: M \rightarrow P$ is a local section around the point $x \in M$.

Unlike $J^{h} P, W^{k, h} P$ is a principal bundle over $M$, and its structure group is

$$
W_{m}^{k, h} G:=G_{m}^{k} \rtimes T_{m}^{h} G .
$$

$W_{m}^{k, h} G$ is called the $(m ; k, h)$-principal prolongation of $G$. The group multiplication on $W_{m}^{k, h} G$ is defined by the following rule:

$$
\left(j_{0}^{k} \alpha, j_{0}^{h} a\right) \odot\left(j_{0}^{k} \beta, j_{0}^{h} b\right):=\left(j_{0}^{k}(\alpha \circ \beta), j_{0}^{h}((a \circ \beta) \cdot b)\right),
$$

'? denoting the group multiplication in $G$. The right action of $W_{m}^{k, h} G$ on $W^{k, h} P$ is then defined by:

$$
\left(j_{0}^{k} \epsilon, j_{x}^{h} \sigma\right) \odot\left(j_{0}^{k} \alpha, j_{0}^{h} a\right):=\left(j_{0}^{k}(\epsilon \circ \alpha), j_{x}^{h}\left(\sigma \cdot\left(a \circ \alpha^{-1} \circ \epsilon^{-1}\right)\right)\right),
$$

$\therefore$ denoting now the canonical right action of $G$ on $P$.

In the case $h=0$, we have a direct product of Lie groups $W_{m}^{k, 0} G:=G_{m}^{k} \times G$ and the usual fibred product $W^{k, 0} P \equiv L^{k} M \times_{M} P$ of principal bundles.

Remark 2.1. When $h=k$, the functor $W^{k, k}$ is often simply written $W^{k}$.

Definition 2.5. Let $\Phi: P \rightarrow P$ be an automorphism over a diffeomorphism $\varphi: M \rightarrow M$ (cf. $\S 1.2)$. We define an automorphism of $W^{k, h} P$ associated with $\Phi$ by

$$
W^{k, h} \Phi: W^{k, h} P \rightarrow W^{k, h} P, \quad\left(j_{0}^{k} \epsilon, j_{x}^{h} \sigma\right) \mapsto\left(j_{0}^{k}(\varphi \circ \epsilon), j_{x}^{h}\left(\Phi \circ \sigma \circ \varphi^{-1}\right)\right) .
$$

Proposition 2.1. The bundle morphism $W^{k, h} \Phi$ preserves the right action, thereby being a principal automorphism.

By virtue of (2.1) and (2.2) $W^{k, h}$ turns out to be a functor from the category of principal $G$-bundles over $m$-dimensional manifolds and local isomorphisms to the category of principal $W_{m}^{k, h} G$-bundles [28]. Now, let $P_{\lambda}:=W^{k, h} P \times_{\lambda} F$ be a fibre bundle associated with $P(M, G)$ via an action $\lambda$ of $W_{m}^{k, h} G$ on a manifold $F$. There exists a canonical representation of the automorphisms of $P$ induced by (2.2). Indeed, if $\Phi: P \rightarrow P$ is an automorphism over a diffeomorphism $\varphi: M \rightarrow M$, then we can define the corresponding induced automorphism $\Phi_{\lambda}$ as

$$
\Phi_{\lambda}: P_{\lambda} \rightarrow P_{\lambda}, \quad \Phi_{\lambda}:[u, f]_{\lambda} \mapsto\left[W^{k, h} \Phi(u), f\right]_{\lambda}
$$

which is well-defined since it turns out to be independent of the representative $(u, f), u \in P$, $f \in F$. This construction yields a functor ${ }_{\lambda}$ from the category of principal $G$-bundles to the category of fibred manifolds and fibre-respecting mappings.

Definition 2.6. A gauge-natural bundle of order $(k, h)$ over $M$ associated with $P(M, G)$ is any such functor.

If we now restrict attention to the case $G=\{e\}$ and $h=0$, we can recover the classical notion of natural bundles over $M$. In particular, we have the following

Definition 2.7. Let $\varphi: M \rightarrow M$ be a diffeomorphism. We define an automorphism of $L^{k} M$ associated with $\varphi$, called its natural lift (onto $L^{k} M$ ), by

$$
L^{k} \varphi: L^{k} M \rightarrow L^{k} M, \quad L^{k} \varphi: j_{0}^{k} \epsilon \mapsto j_{0}^{k}(\varphi \circ \epsilon) .
$$


Then, $L^{k}$ turns out to be a functor from the category of $m$-dimensional manifolds and local diffeomorphisms to the category of principal $G_{m}^{k}$-bundles. Now, given any fibre bundle associated with $L^{k} M$ and any diffeomorphism on $M$, we can define a corresponding induced automorphism in the usual fashion. This construction yields a functor from the category of $m$-dimensional manifolds to the category of fibred manifolds.

Definition 2.8. A natural bundle of order $k$ over $M$ is any such functor.

Remark 2.2. In the sequel we shall always assume that $L^{k} M$ is equipped with the principal bundle structure naturally induced by the differentiable structure of the base manifold $M$, i.e. that $L^{k} M$ itself is a natural bundle over $M$. This is, of course, possible because we can always identify a principal bundle $P(M, G)$ with its associated bundle $P_{\lambda}:=P \times_{\lambda} G$, where $\lambda$ is the left action of $G$ on itself.

Now, if $\Xi$ is a $G$-invariant vector field on $P$ generating a one-parameter group $\left\{\Phi_{t}\right\}$ of automorphisms of $P$ and projecting on a vector field $\xi$ on $M$ (cf. §1.2), we can define the induced vector field $\Xi_{\lambda}$ on $P_{\lambda}$ simply by setting

$$
\Xi_{\lambda}:=\left.\frac{\partial}{\partial t}\left(\Phi_{t}\right)_{\lambda}\right|_{t=0},
$$

which obviously projects on the same vector field $\xi$ on $M$. Now, on relying on the general theory of Lie derivatives $[44,24,28,20]$, we can give the following

Definition 2.9. Let $P_{\lambda}$ be a gauge-natural bundle associated with some principal bundle $P(M, G)$, $\Xi$ a $G$-invariant vector field on $P$ projecting on a vector field $\xi$ on $M$, and $\sigma: M \rightarrow P_{\lambda}$ a section of $P_{\lambda}$. Then, by the generalized (gauge-natural) Lie derivative of $\sigma$ with respect to $\Xi$ we shall mean the map

$$
£_{\Xi} \sigma: M \rightarrow V P_{\lambda}, \quad £_{\Xi} \sigma:=T \sigma \circ \xi-\Xi_{\lambda} \circ \sigma,
$$

$V P_{\lambda}$ denoting the vertical (tangent) bundle of $P_{\lambda}$.

Definition 2.9 is the conceptually natural generalization of the classical notion of a Lie derivative [48], to which it suitably reduces when applied to natural objects. The "formal" counterpart of $£_{\Xi} \sigma$ is defined in the usual way.

Definition 2.10. Let $P_{\lambda}, \Xi$ and $\sigma$ be as above. We call formal generalized Lie derivative the global bundle morphism $£_{\Xi y:} J^{1} P_{\lambda} \rightarrow V P_{\lambda}$ intrinsically defined as

$$
£_{\Xi} y \circ j^{1} \sigma=£_{\Xi} \sigma,
$$

where $\Xi_{\Xi} \sigma$ is the Lie derivative of $\sigma$ in the sense of Definition 2.9.

Of course, if $P_{\lambda}$ is a gauge-natural vector or affine bundle (which is always the case in classical field theory), then its vertical bundle splits as $V P_{\lambda} \cong P_{\lambda} \times_{M} P_{\lambda}$ or $V P_{\lambda} \cong P_{\lambda} \times_{M} \vec{P}_{\lambda}$, respectively, $\vec{P}_{\lambda}$ being the vector bundle on which the affine bundle $P_{\lambda}$ is modelled. In this case, we can give the following

Definition 2.11. Let $P_{\lambda}$ be a gauge-natural vector or affine bundle associated with some principal bundle $P(M, G)$, and let $\Xi$ and $\sigma$ be as in Definition 2.9. Then, by the (restricted gauge-natural) Lie derivative of $\sigma$ with respect to $\Xi$ we shall mean the second component of $\Xi_{\Xi} \sigma$. Analogously, we shall call formal (restricted) Lie derivative the second component of $£ \Xi y$.

In the sequel, we shall not formally distinguish between generalized and restricted Lie derivatives, as it should be clear from the context which is the operator under actual consideration. 


\section{$3 \quad$ Noether theorem and conserved quantities}

Let $M$ be an $m$-dimensional (orientable) manifold. By a $k$-th order Lagrangian on a gauge-natural bundle $P_{\lambda}$, associated with some principal bundle $P(M, G)$, we shall mean a base-preserving morphism

$$
\mathcal{L}: J^{k} P_{\lambda} \rightarrow \bigwedge^{m} T^{*} M
$$

or, equivalently, a horizontal $m$-form $\mathcal{L} \in \Omega_{0}^{m}\left(J^{k} P_{\lambda}\right)$. Locally, $\mathcal{L}\left(j^{k} y\right)$ reads

$$
\mathcal{L}\left(x^{\lambda}, y^{\mathfrak{a}}{ }_{\boldsymbol{\mu}}\right)=L\left(x^{\lambda}, y^{\mathfrak{a}}{ }_{\boldsymbol{\mu}}\right) \mathrm{d} s
$$

for some scalar density $L$ on $J^{k} P_{\lambda}, \mathrm{d} s \equiv \mathrm{d} x^{0} \wedge \mathrm{d} x^{1} \wedge \cdots \wedge \mathrm{d} x^{m-1}$ denoting the local volume element on $M$. Then we have the following result (cf., e.g., [28]).

Proposition 3.1. There exist a global morphism $\mathbb{F}(\mathcal{L}): J^{2 k-1} P_{\lambda} \rightarrow V^{*} J^{k-1} P_{\lambda} \otimes \bigwedge^{m-1} T^{*} M$ and a unique global morphism $\mathbb{E}(\mathcal{L}): J^{2 k} P_{\lambda} \rightarrow V^{*} P_{\lambda} \otimes \bigwedge^{m} T^{*} M$ such that

$$
\left(\pi_{k}^{2 k}\right)^{*}\left\langle\mathrm{~d} \mathcal{L}, J^{k} \Upsilon\right\rangle=\langle\mathbb{E}(\mathcal{L}), \Upsilon\rangle+\mathrm{d}_{\mathrm{H}}\left\langle\mathbb{F}(\mathcal{L}), J^{k-1} \Upsilon\right\rangle
$$

for any vertical vector field $\Upsilon$ on $P_{\lambda} \cdot{ }^{2}$ Locally,

$$
\mathbb{E}(\mathcal{L})=\left(\frac{\partial L}{\partial y^{\mathfrak{a}}}+\sum_{1 \leqslant|\boldsymbol{\mu}| \leqslant k}(-1)^{|\boldsymbol{\mu}|} \mathrm{d}_{\boldsymbol{\mu}} \frac{\partial L}{\partial y^{\mathfrak{a}} \boldsymbol{\mu}}\right) \overline{\mathrm{d}} y^{\mathfrak{a}} \otimes \mathrm{d} s,
$$

$\left\{\overline{\mathrm{d}} y^{\mathfrak{a}}\right\}$ denoting the fibre basis of $V^{*} P_{\lambda}:=\left(V P_{\lambda}\right)^{*}$ defined by requiring $\left\langle\overline{\mathrm{d}} y^{\mathfrak{a}}, \partial_{\mathfrak{b}}\right\rangle=\delta^{\mathfrak{a}}{ }_{\mathfrak{b}}$.

Classically, $\left(\pi_{k}^{2 k}\right)^{*}\left\langle\mathrm{~d} \mathcal{L}, J^{k} \Upsilon\right\rangle$ is denoted by $\delta \mathcal{L}$, a notation we shall use ourselves when there is no danger of confusion, and identity $(3.2)$ is known as the first variation formula. $\mathbb{E}(\mathcal{L})$ is called the Euler-Lagrange morphism, while $\mathbb{F}(\mathcal{L})$ is known as the Poincaré-Cartan morphism. It is not uniquely determined and depends in general on a linear connection on $M$. In the case $k=1,2$, though, this dependence disappears and $\mathbb{F}(\mathcal{L})$ locally reads

$$
\mathbb{F}(\mathcal{L})=\left[\left(\frac{\partial L}{\partial y^{\mathfrak{a}}{ }_{\mu}}-(k-1) \mathrm{d}_{\nu} \frac{\partial L}{\partial y^{\mathfrak{a}}{ }_{\mu \nu}}\right) \overline{\mathrm{d}} y^{\mathfrak{a}}+(k-1) \frac{\partial L}{\partial y^{\mathfrak{a}}{ }_{\mu \nu}} \overline{\mathrm{d}} y^{\mathfrak{a}}{ }_{\nu}\right] \otimes \mathrm{d} s_{\mu},
$$

where $\mathrm{d} s_{\mu}$ stands for $\left.\partial_{\mu}\right\lrcorner \mathrm{d} s$ and $\overline{\mathrm{d}} y^{\mathfrak{a}}{ }_{\nu}$ is defined by requiring $\left\langle\overline{\mathrm{d}} y^{\mathfrak{a}}{ }_{\nu}, \partial_{\mathfrak{b}}{ }^{\rho}\right\rangle=\delta^{\mathfrak{a}}{ }_{\mathfrak{b}} \delta^{\rho}{ }_{\nu}$.

Definition 3.1. A configuration bundle automorphism $\left(\varphi, \Phi_{\lambda}\right)$, i.e. an automorphism $\Phi_{\lambda}$ of $P_{\lambda}$ induced by an automorphism $\Phi$ of $P$ over a diffeomorphism $\varphi: M \rightarrow M$, is called a (Lagrangian) symmetry for Lagrangian (3.1) if it leaves $\mathcal{L}$ unchanged, i.e. if $\bigwedge^{m} T^{*} \varphi \circ \mathcal{L} \circ j^{1} \Phi_{\lambda}=\mathcal{L}$.

Clearly, the infinitesimal version of Definition 3.1 is the following.

Definition 3.2. A vector field $\Xi_{\lambda}$ generating a one-parameter group $\left\{\left(\Phi_{t}\right)_{\lambda}\right\}$ of symmetries is called an infinitesimal (Lagrangian) symmetry.

Definition 3.3. Let $\operatorname{Aut}\left(P_{\lambda}\right)$ denote the group of all induced automorphisms of $P_{\lambda}$. We shall say that a $k$-th order Lagrangian on $P_{\lambda}$ is $\operatorname{Aut}\left(P_{\lambda}\right)$-invariant if any induced automorphism of $P_{\lambda}$ is a symmetry (and any induced vector field on $P_{\lambda}$ is an infinitesimal symmetry).

Definition 3.4. A $k$-th order Lagrangian field theory on a gauge-natural bundle $P_{\lambda}$ is a field theory where the fields are represented by (local) sections of $P_{\lambda}$ and the equations of motion can be formally written as

$$
\mathbb{E}(\mathcal{L}) \circ j^{2 k} \sigma=0
$$

\footnotetext{
${ }^{2}$ The use of the horizontal differential in (3.2) is justified by the one-to-one correspondence between horizontal forms and base-preserving morphisms mentioned in $\S 1.1$.
} 
for some suitable Aut $\left(P_{\lambda}\right)$-invariant $k$-th order Lagrangian $\mathcal{L}$ on $P_{\lambda}$ and some section $\sigma \in \Gamma\left(P_{\lambda}\right)$. $P_{\lambda}$ is called the configuration bundle of the theory, $P$ its structure bundle and $\sigma$ a critical section of $P_{\lambda}$, whereas (the local expression of) equation (3.3) is known as the Euler-Lagrange equations. Whenever an identity holds only modulo equation (3.3), we shall say that it holds "on shell" and use the symbol ' $\approx$ ' instead of the equals sign. In particular, we shall write equation (3.3) itself simply as $\mathbb{E}(\mathcal{L}) \approx 0$.

All known classical Lagrangian field theories such as classical mechanics, elasticity, gravitational field theories (including, in particular, Einstein's general relativity and the Einstein-Cartan theory), electromagnetism, the Yang-Mills theory, bosonic and fermionic matter field theories, topological field theories - as well as all their possible mutual couplings - are Lagrangian field theories on some suitable gauge-natural (vector or affine) bundle [7, 28, 10].

Proposition 3.2. Let $\Xi_{\lambda}$ be a vector field on $P_{\lambda}$ induced by a $G$-invariant vector field $\Xi$ on $P$ projecting on a vector field $\xi$ on $M$, and $\mathcal{L}$ an $\operatorname{Aut}\left(P_{\lambda}\right)$-invariant $k$-th order Lagrangian on $P_{\lambda}$. Then,

$$
\left.\left\langle\mathrm{d} \mathcal{L}, J^{k} £_{\Xi} y\right\rangle=\mathrm{d}_{\mathrm{H}}(\xi\lrcorner \mathcal{L}\right) .
$$

Proof. The result readily follows from Definition 3.2 and the properties of the formal Lie derivative, taking into account the isomorphism $J^{k} V P_{\lambda} \cong V J^{k} P_{\lambda}$.

Identity (3.4) is known as the fundamental identity. Combining (3.2) and (3.4) we get

$$
\mathrm{d}_{\mathrm{H}} E(\mathcal{L}, \Xi)=W(\mathcal{L}, \Xi),
$$

where we set

$$
\left.E(\mathcal{L}, \Xi):=\left\langle\mathbb{F}(\mathcal{L}), J^{k-1} £_{\Xi} y\right\rangle-\xi\right\lrcorner \mathcal{L}
$$

and

$$
W(\mathcal{L}, \Xi):=-\left\langle\mathbb{E}(\mathcal{L}), £_{\Xi} y\right\rangle .
$$

$E(\mathcal{L}, \Xi)$ is called the Noether current and $W(\mathcal{L}, \Xi)$ the work form. Formula $(3.5)$ is the generalization of Noether's theorem [34] to the geometric framework of jet prolongations of gauge-natural bundles. Indeed, if we define

$$
\begin{aligned}
E(\mathcal{L}, \Xi, \sigma) & :=\left(j^{2 k-1} \sigma\right)^{*} E(\mathcal{L}, \Xi), \\
W(\mathcal{L}, \Xi, \sigma) & :=\left(j^{2 k} \sigma\right)^{*} W(\mathcal{L}, \Xi),
\end{aligned}
$$

we have

$$
\mathrm{d} E(\mathcal{L}, \Xi, \sigma)=W(\mathcal{L}, \Xi, \sigma)
$$

and, whenever $\sigma$ is a critical section,

$$
\mathrm{d} E(\mathcal{L}, \Xi, \sigma)=0 .
$$

Thus, given an infinitesimal Lagrangian symmetry $\Xi$, we have a whole class of currents $E(\mathcal{L}, \Xi, \sigma)$ (one for each solution $\sigma$ ), which are closed $(m-1)$-forms on $M$. We stress that the Noether current $E(\mathcal{L}, \Xi)$ is defined at the bundle level and is canonically associated with the Lagrangian $\mathcal{L}$. It is only at a later stage that it is evaluated on a section $\sigma: M \rightarrow P_{\lambda}$, thereby giving $E(\mathcal{L}, \Xi, \sigma)$.

Since $E(\mathcal{L}, \Xi, \sigma)$ is an $(m-1)$-form on $M$, it can be integrated over an $(m-1)$-dimensional region $\Sigma$, namely a compact submanifold $\Sigma \hookrightarrow M$ with boundary $\partial \Sigma$.

Definition 3.5. The real functional

$$
Q_{\Sigma}(\mathcal{L}, \Xi, \sigma):=\int_{\Sigma} E(\mathcal{L}, \Xi, \sigma)
$$

is called the conserved quantity (or charge) along $\sigma$ with respect to the infinitesimal symmetry $\Xi$ and the region $\Sigma$. 
Indeed, if $\sigma$ is a critical section, and two compact $(m-1)$-submanifolds $\Sigma, \Sigma^{\prime} \hookrightarrow M$ form the homological boundary $\partial D$ of a compact $m$-dimensional domain $D \subseteq M$, from (3.8), Stokes's theorem and (3.7) we readily obtain

$$
Q_{\Sigma^{\prime}}(\mathcal{L}, \Xi, \sigma)=Q_{\Sigma}(\mathcal{L}, \Xi, \sigma) .
$$

Since $E(\mathcal{L}, \Xi, \sigma)$ is closed on shell, in field theories where $m>1$ we may ask ourselves whether it is also exact, i.e. whether there exists an $(m-2)$-form $U(\mathcal{L}, \Xi, \sigma)$ on $M$ such that

$$
E(\mathcal{L}, \Xi, \sigma)=\mathrm{d} U(\mathcal{L}, \Xi, \sigma) .
$$

If this happens to be the case, then we can express $Q_{\Sigma}(\mathcal{L}, \Xi, \sigma)$ as an $(m-2)$-dimensional integral over the boundary $\partial \Sigma$ of $\Sigma$. Indeed, on using (3.9) and Stokes's theorem, we have

$$
Q_{\Sigma}(\mathcal{L}, \Xi, \sigma)=\int_{\partial \Sigma} U(\mathcal{L}, \Xi, \sigma) .
$$

Actually, it is possible to prove the following fundamental

Theorem 3.1. The Noether current is exact on shell for all gauge-natural field theories, regardless of the topology of $M[10]^{3}$.

We stress that this important result can only be achieved since Noether's theorem has been formulated in terms of fibred morphisms rather than directly in terms of forms on $M$. Notably, we shall give the following

Definition 3.6. If the Noether current $E(\mathcal{L}, \Xi)$ can be written as

$$
E(\mathcal{L}, \Xi)=\tilde{E}(\mathcal{L}, \Xi)+\mathrm{d}_{\mathrm{H}} U(\mathcal{L}, \Xi),
$$

where $\tilde{E}(\mathcal{L}, \Xi, \sigma):=\left(j^{2 k-1} \sigma\right)^{*} \tilde{E}(\mathcal{L}, \Xi)$ vanishes for any critical section $\sigma$, then we shall call $\tilde{E}(\mathcal{L}, \Xi)$ and $U(\mathcal{L}, \Xi)$ the reduced current and the superpotential associated with $\mathcal{L}$, respectively. Whenever the splitting (3.11) holds, then it is immediate to see that $U(\mathcal{L}, \Xi, \sigma):=\left(j^{2 k-1} \sigma\right)^{*} U(\mathcal{L}, \Xi)$ satisfies equation (3.9) for any critical section $\sigma$.

Of course, neither Noether currents nor superpotentials are unique: the former are defined modulo exact $(m-1)$-forms, the latter modulo closed $(m-2)$-forms. What is physically meaningful, though, are the conserved quantities, which only depend on the cohomology class, not on the chosen representative.

Finally, one might be interested in what happens to Noether currents and superpotentials (and, hence, to the conserved quantities) when the Lagrangian of the theory is replaced by an equivalent one. We recall that two Lagrangians $\mathcal{L}$ and $\mathcal{L}^{\prime}$ are said to be equivalent if $\mathbb{E}(\mathcal{L})=\mathbb{E}\left(\mathcal{L}^{\prime}\right)$. Owing to linearity, this is tantamount to saying that two Lagrangians are equivalent if they differ from each other by a (variationally) trivial Lagrangian, i.e. a Lagrangian whose Euler-Lagrange morphism is identically zero. The issue of finding all trivial Lagrangians represents one of the most difficult problems of the geometric calculus of variations and, in the $k$-th order case, was only recently solved by Krupka and Musilová [30]. In the present context, their result can be rephrased as follows.

Theorem 3.2. Two $k$-th order Lagrangians $\mathcal{L}$ and $\mathcal{L}^{\prime}$ on a gauge-natural bundle $P_{\lambda}$ are equivalent iff, locally, they differ from each other by the horizontal part of the exterior differential of an $(m-1)$-form $\chi$ on $J^{k-1} P_{\lambda}$.

Therefore, if $\mathcal{L}^{\prime}:=\mathcal{L}+h(\mathrm{~d} \chi)$ and we set $\beta:=h(\chi)$, we readily find

$$
\left.E\left(\mathcal{L}^{\prime}, \Xi\right)=E(\mathcal{L}, \Xi)+£_{J^{k}} \beta-\xi\right\lrcorner \mathrm{d}_{\mathrm{H}} \beta .
$$

But $\beta \in \Omega_{0}^{m-1}\left(J^{k} P_{\lambda}\right)$; accordingly, $\left.\left.£_{J^{k} \Xi} \beta=£_{\xi} \beta \equiv \xi\right\lrcorner \mathrm{d}_{\mathrm{H}} \beta+\mathrm{d}_{\mathrm{H}}(\xi\lrcorner \beta\right)$, whence

$$
\begin{aligned}
& \tilde{E}\left(\mathcal{L}^{\prime}, \Xi\right)=\tilde{E}(\mathcal{L}, \Xi), \\
& \left.U\left(\mathcal{L}^{\prime}, \Xi\right)=U(\mathcal{L}, \Xi)+\xi\right\lrcorner \beta .
\end{aligned}
$$

\footnotetext{
${ }^{3}$ The proof closely follows the analogous one for natural field theories due to Robutti [37] (see also [17, 18]).
} 


\section{Spin-tetrads, spin-connections and spinors}

To the best of our knowledge, the concept of a "free spin structure" was originally introduced (with a different purpose) in [36] (see also [42]). It was then rediscovered in [46] for the very reason mentioned in the Introduction and further analysed in $[12,13]$. The notion of a "spin-tetrad" as a section of a suitable gauge-natural bundle over $M$ was first proposed in [12].

Definition 4.1. Let $M$ be a 4-dimensional manifold admitting Lorentzian metrics of signature -2 , i.e. satisfying the topological requirements which ensure the existence on it of Lorentzian structures $\left[\mathrm{SO}(1,3)^{e}\right.$-reductions], and let $\Lambda$ be the epimorphism which exhibits $\operatorname{Spin}(1,3)^{e}$ as the twofold covering of $\mathrm{SO}(1,3)^{e}$. A free spin structure on $M$ consists of a principal bundle $\pi: \Sigma \rightarrow M$ with structure group $\operatorname{Spin}(1,3)^{e}$ and a map $\tilde{\Lambda}: \Sigma \rightarrow L M$ such that

$$
\begin{gathered}
\tilde{\Lambda} \circ r^{S}=r^{(\llcorner\circ \Lambda)(S)} \circ \tilde{\Lambda} \quad \forall S \in \operatorname{Spin}(1,3)^{e}, \\
\pi^{\prime} \circ \tilde{\Lambda}=\pi,
\end{gathered}
$$

$r$ and $r^{\prime}$ denoting the canonical right actions on $\Sigma$ and $L M$, respectively, $\iota: \mathrm{SO}(1,3)^{e} \rightarrow \mathrm{GL}(4, \mathbb{R})$ the canonical injection of Lie groups, and $\pi^{\prime}: L M \rightarrow M$ the canonical projection. We shall call the bundle map $\tilde{\Lambda}$ a spin-frame on $\Sigma$.

This definition of a spin structure induces metrics on $M$. Indeed, given a spin-frame $\tilde{\Lambda}: \Sigma \rightarrow L M$, we can define a metric $g$ via the reduced subbundle $\operatorname{SO}(M, g):=\tilde{\Lambda}(\Sigma)$ of $L M$. In other words, the (dynamic) metric $g \equiv g_{\tilde{\Lambda}}$ is defined to be the metric such that frames in $\tilde{\Lambda}(\Sigma) \subset L M$ are $g$-orthonormal frames. It is important to stress that in our picture the metric $g$ is built up a posteriori, after a spin-frame has been determined by the field equations in a way which is compatible with the (free) spin structure one has used to define spinors.

Now, if we want to regard spin-frames as dynamical variables in a Lagrangian field theory, we should be able to represent them as (global) sections of a suitable configuration bundle. This motivates the following

Definition 4.2. Let $\Lambda$ be as in Definition 4.1 and consider the following left action of the group $W_{4}^{1,0} \operatorname{Spin}(1,3)^{e}$ on the manifold $\mathrm{GL}(4, \mathbb{R})$

$$
\rho:((A, S), \theta) \mapsto \theta^{\prime}:=\Lambda(S) \circ \theta \circ A^{-1}
$$

together with the associated bundle $\Sigma_{\rho}:=W^{1,0} \Sigma \times_{\rho} \operatorname{GL}(4, \mathbb{R}) . \Sigma_{\rho}$ is a fibre bundle associated with $W^{1,0} \Sigma$, i.e. a gauge-natural bundle of order $(1,0)$. A section of $\Sigma_{\rho}$ will be called a spin-tetrad.

If $\left(\theta_{\mu}^{a}\right)$ denote the components of a spin-tetrad $\theta$ in some local chart, then the components $\left(g_{\mu \nu}\right)$ of the induced metric $g$ in the associated chart read

$$
g_{\mu \nu} \equiv \theta^{a}{ }_{\mu} \theta^{b}{ }_{\nu} \eta_{a b},
$$

formally identical with equation (i) of the Introduction, but with both (iii) and (iv) built in.

Recall now that a (principal) connection on a principal bundle $P(M, G)$ may be regarded as a $G$-equivariant global section of the affine jet bundle $J^{1} P \rightarrow P$, where the $G$-action on $J^{1} P$ is induced by the first jet prolongation of the canonical (right) action of $G$ on $P$. Owing to $G$-equivariance there is a one-to-one correspondence between principal connections and global sections of the quotient bundle $J^{1} P / G \rightarrow M(c f .[28,19])$. Bearing this in mind, we can give

Definition 4.3. Let $\mathfrak{s o}(1,3) \cong \mathfrak{s p i n}(1,3)$ denote the Lie algebra of $\operatorname{SO}(1,3)^{e}$ and consider the following left action of the group $W_{4}^{1,1} \operatorname{Spin}(1,3)^{e} \cong \mathrm{GL}(4, \mathbb{R}) \times \operatorname{Spin}(1,3)^{e} \rtimes\left(\left(\mathbb{R}^{4}\right)^{*} \otimes \mathfrak{s o}(1,3)\right)$ on the vector space $\left(\mathbb{R}^{4}\right)^{*} \otimes \mathfrak{s o}(1,3)$

$$
\ell:((A, S, \hat{S}), \omega) \mapsto \omega^{\prime}:=\operatorname{Ad}_{\Lambda(S)}(\omega-\hat{\Lambda}(\hat{S})) \circ A^{-1},
$$

where Ad denotes the adjoint representation of $\mathrm{SO}(1,3)^{e}$, and $\hat{\Lambda}:=\operatorname{id} \otimes T_{e} \Lambda$ the isomorphism between $\left(\mathbb{R}^{4}\right)^{*} \otimes \mathfrak{s p i n}(1,3)$ and $\left(\mathbb{R}^{4}\right)^{*} \otimes \mathfrak{s o}(1,3)$ induced by $\Lambda$. Clearly, the associated bundle $\Sigma_{\ell}:=W^{1,1} \Sigma \times_{\ell}\left(\left(\mathbb{R}^{4}\right)^{*} \otimes \mathfrak{s o}(1,3)\right)$ is a gauge-natural bundle of order $(1,1)$ isomorphic to $J^{1}\left(\Sigma / \mathbb{Z}_{2}\right) / \mathrm{SO}(1,3)^{e}$. A section of $\Sigma_{\ell}$ will be called a spin-connection. 
Note that also spinors can be regarded as sections of a suitable gauge-natural bundle over $M$. Indeed, if $\hat{\gamma}$ is the linear representation of $\operatorname{Spin}(1,3)^{e}$ on the vector space $\mathbb{C}^{4}$ induced by the given choice of $\gamma$ matrices, then the associated vector bundle $\Sigma_{\hat{\gamma}}:=\Sigma \times_{\hat{\gamma}} \mathbb{C}^{4}$ is a gauge-natural bundle of order $(0,0)$ whose sections represent spinors (or, more precisely, spin-vector fields). Therefore, in spite of what is usually believed, a Lie derivative for spinors (in the sense of Definition 2.9) always exists, no matter what the vector field $\xi$ on $M$ is $(c f .[11,20])$.

Finally, for the sake of completeness, we shall recall that the Dirac adjoint $\bar{\psi}$ of a spinor $\psi$ is defined as the adjoint of $\psi$ with respect to the standard $\operatorname{Spin}(1,3)^{e}$-invariant scalar product on $\mathbb{C}^{4}$ (see, e.g., [5]). With our conventions, $\bar{\psi}$ locally reads

$$
\bar{\psi}(x)=\psi^{\dagger}(x) \gamma_{0}
$$

for all $x \in M$. Note also that, in our picture, the spinor connection $\tilde{\omega}$ corresponding to a given spin-connection $\omega$ may be defined in terms of $\omega$ as

$$
\tilde{\omega}:=\left(\mathrm{id} \otimes\left(\Lambda^{\prime}\right)^{-1}\right)(\omega),
$$

$\Lambda^{\prime}:=T_{e} \Lambda$ denoting the Lie algebra isomorphism between $\mathfrak{s p i n}(1,3)$ and $\mathfrak{s o}(1,3)$. On differentiating (1.4a) and taking (1.4b) into account, we find that $\left(\Lambda^{\prime}\right)^{-1}(\mathfrak{l})$ is given by ${ }^{4}$

$$
\left(\Lambda^{\prime}\right)^{-1}(\mathfrak{l}) \equiv-\frac{1}{4} \mathfrak{l}^{a b} \gamma_{a b}
$$

for all $\mathfrak{l} \equiv\left(\mathfrak{l}_{c}^{a}=: \mathfrak{l}^{a b} \eta_{b c}\right) \in \mathfrak{s o}(1,3)$. Therefore, the components $\left(\tilde{\omega}_{\mu}\right)$ of $\tilde{\omega}$ read

$$
\tilde{\omega}_{\mu} \equiv-\frac{1}{4} \omega^{a b}{ }_{\mu} \gamma_{a b}
$$

$\left(\omega^{a}{ }_{c \mu}=: \omega^{a b}{ }_{\mu} \eta_{b c}\right)$ denoting the components of $\omega$. Identity (4.2) is what is used in practice for evaluating the covariant derivative of a spinor and its Dirac adjoint,

$$
\begin{aligned}
& \nabla_{\mu} \psi:=\partial_{\mu} \psi+\tilde{\omega}_{\mu} \psi, \\
& \nabla_{\mu} \bar{\psi}=\overline{\nabla_{\mu} \psi} \equiv \partial_{\mu} \bar{\psi}-\bar{\psi} \tilde{\omega}_{\mu} .
\end{aligned}
$$

On making use of (2.4) we can now readily evaluate the Lie derivatives, with respect to a $\operatorname{Spin}(1,3)^{e}$-invariant vector field $\Xi$ on $\Sigma$, of a spin-tetrad, a spin-connection, a spinor and its Dirac adjoint, which will locally read

$$
\begin{aligned}
£_{\Xi} \theta^{a}{ }_{\mu} & =\xi^{\nu} \partial_{\nu} \theta^{a}{ }_{\mu}+\partial_{\mu} \xi^{\nu} \theta^{a}{ }_{\nu}-\Xi_{b}^{a} \theta_{\mu}{ }_{\mu}, \\
£_{\Xi} \omega_{b \mu}^{a} & =\xi^{\nu} \partial_{\nu} \omega^{a}{ }_{b \mu}+\partial_{\mu} \xi^{\nu} \omega^{a}{ }_{b \nu}+\omega^{a}{ }_{c \mu} \Xi_{b}^{c}-\omega_{b \mu}^{c} \Xi_{c}^{a}+\partial_{\mu} \Xi_{b}^{a}, \\
£_{\Xi} \psi & =\xi^{\nu} \partial_{\nu} \psi+\frac{1}{4} \Xi^{a b} \gamma_{a b} \psi, \\
£_{\Xi} \bar{\psi} & =\overline{£_{\Xi} \psi} \equiv \xi^{\nu} \partial_{\nu} \bar{\psi}-\frac{1}{4} \Xi^{a b} \bar{\psi} \gamma_{a b},
\end{aligned}
$$

respectively, $\left(\xi^{\mu}, \Xi^{a}{ }_{c}=: \Xi^{a b} \eta_{b c}\right)$ denoting the components of the $\mathrm{SO}(1,3)^{e}$-invariant vector field $\Xi_{\tilde{\Lambda}}$ induced by $\Xi$.

\section{Einstein (-Cartan) -Dirac theory}

Throughout this section we shall use Cartan's language of vector (bundle)-valued differential forms (on $M$ ), which will prove to be an elegant and compact way to express our findings. To this end, let $\Sigma_{\hat{\rho}}:=\Sigma \times_{\hat{\rho}} \mathbb{R}^{4}$ denote the vector bundle associated with $\Sigma$ via the action

$$
\hat{\rho}: \operatorname{Spin}(1,3)^{e} \times \mathbb{R}^{4} \rightarrow \mathbb{R}^{4}, \quad(S, u) \mapsto \Lambda(S) \circ u .
$$

\footnotetext{
${ }^{4}$ Here and in the sequel, $\mathfrak{s o}(1,3)$ is understood to be represented on the Lie algebra of $4 \times 4$ real matrices by means of its "fundamental representation", i.e. its lowest dimensional faithful (linear) representation.
} 
Then, a spin-tetrad can be equivalently regarded as a $\Sigma_{\hat{\rho}}$-valued 1-form on $M$ locally reading

$$
\theta:=\theta^{a} \otimes f_{a}, \quad \theta^{a}:=\theta^{a}{ }_{\mu} \mathrm{d} x^{\mu},
$$

$\left(f_{a}\right)$ denoting a local fibre basis of $\Sigma_{\hat{\rho}}$. Furthermore, let $\mathfrak{g l}\left(\Sigma_{\hat{\rho}}\right)$ denote the vector bundle over $M$ given by the value at $\Sigma_{\hat{\rho}}$ of the canonical extension of the functor $\mathfrak{g l}$ to the category of vector bundles and their homomorphisms (see [28, §6.7]). Finally, if $\left(\omega^{a}{ }_{b \mu}\right)$ are the components of a spin-connection in some local chart, it is convenient to introduce the notation

$$
\omega_{b}^{a}:=\omega_{b \mu}^{a} \mathrm{~d} x^{\mu} .
$$

\subsection{Riemann-Cartan geometry on spin manifolds}

Now, let ' $\nabla$ ' be the covariant derivative operator with respect to the connection on $T_{q}^{p} M$ naturally induced by a linear connection $\Gamma$ on $L M, T_{q}^{p} M$ denoting the $(p, q)$-tensor bundle over $M$. Classically, Riemann-Cartan geometry is characterized by two conditions: the covariant constancy of the metric,

$$
\nabla g=0,
$$

just as in ordinary Riemannian geometry, and the presence of a (not necessarily zero) torsion tensor $\tau$ such that

$$
\tau\left(\xi, \xi^{\prime}\right)=\nabla_{\xi} \xi^{\prime}-\nabla_{\xi^{\prime}} \xi-\left[\xi, \xi^{\prime}\right]
$$

for any two vector fields $\xi$ and $\xi^{\prime}$ on $M$.

In the present gauge-natural setting we can introduce analogous concepts serving a similar purpose. In particular, if $\theta$ is a spin-tetrad in the sense of Definition 4.2 and $g$ is the metric induced by $\theta$ via (4.1), equation (5.2) can be derived by the condition

$$
\nabla \theta=0,
$$

where, here, ' $\nabla$ ' denotes the covariant derivative operator with respect to the connection on $\Sigma_{\rho}$ canonically induced by the connections $\Gamma$ and $\tilde{\omega}$ on $L M$ and $\Sigma$, respectively (see also §5.2.1). Accordingly, we can define a torsion 2 -form as the $\Sigma_{\hat{\rho}}$-valued 2-form, which we shall denote again by $\tau$, given by the expression

$$
\tau:=\mathcal{D} \theta
$$

or, equivalently,

$$
\tau:=\tau^{a} \otimes f_{a}, \quad \tau^{a}:=\mathcal{D} \theta^{a} \equiv \mathrm{d} \theta^{a}+\omega^{a}{ }_{b} \wedge \theta^{b},
$$

' $\mathcal{D}$ ' denoting the "covariant exterior derivative" operator $[28, \S 11.13$ et seq. $]$ and $\theta$ being as in (5.1). Moreover, we can define a contortion 1 -form as the $\mathfrak{g l}\left(\Sigma_{\hat{\rho}}\right)$-valued 1 -form measuring the deviation of the spin-connection $\omega$ from the Riemannian (or "Levi-Civita") spin-connection ${ }^{\theta} \omega$ [see (5.6) below]:

$$
K:=\left(\omega^{a}{ }_{b}-{ }^{\theta} \omega_{b}^{a}\right) \otimes F_{a}^{b},
$$

$\left(F_{a}{ }^{b}\right)$ denoting a local fibre basis of $\mathfrak{g l}\left(\Sigma_{\hat{\rho}}\right)$. The components of the associated tensor field then read

$$
K^{a b c}=-\frac{1}{2}\left(\tau^{a b c}+\tau^{b c a}-\tau^{c a b}\right),
$$

$\left(\tau_{d e}^{a}=: \tau^{a b c} \eta_{b d} \eta_{c e}\right)$ denoting the components of the tensor associated to the torsion 2-form. Finally, note that a curvature 2 -form associated with $\omega$ may be defined as the $\mathfrak{g l}\left(\Sigma_{\hat{\rho}}\right)$-valued 2 -form

$$
\Omega:=\Omega_{b}^{a} \otimes F_{a}^{b}, \quad \Omega_{b}^{a}:=\mathrm{d} \omega_{b}^{a}+\omega^{a}{ }_{c} \wedge \omega_{b}^{c},
$$

and that the components of the Riemannian spin-connection ${ }^{\theta} \omega$ read (cf. [6])

$$
{ }^{\theta} \omega^{a b}{ }_{\mu}=\theta^{b \nu} \partial_{[\nu} \theta^{a}{ }_{\mu]}+\theta^{a \rho} \theta_{c \mu} \theta^{b \nu} \partial_{[\nu} \theta_{\rho]}^{c}+\theta^{a \nu} \partial_{[\mu} \theta_{\nu]}^{b} \equiv{ }^{\theta} \omega^{[a b]}{ }_{\mu},
$$

Latin and Greek indices being lowered or raised by $\eta$ and $g$, respectively, or their inverses. 
We are now in a position to apply the theory of conserved quantities developed in $\S 3$ to the Einstein (-Cartan) -Dirac theory. We shall do so separately for the Einstein-Cartan-Dirac case and the Einstein-Dirac one. Calculations will be "formal", unless otherwise stated, i.e. they will involve local coordinates, rather than sections, of the bundles under consideration. For the sake of simplicity, we shall nevertheless use the names of the corresponding sections. With a slight abuse of notation, we shall also use the symbols ' $\nabla$ ' and ' $\mathcal{D}$ ' for their formal counterparts, defined in the usual manner ( $c f$. . e.g., Definition 2.10).

\subsection{Einstein-Cartan-Dirac theory}

Our main reference for the Einstein-Cartan-Dirac theory is [6].

In the light of the new geometric framework developed in $\S 4$, the Einstein-Cartan Lagrangian can be defined as the base-preserving morphism

$$
\mathcal{L}_{\mathrm{EC}}: \Sigma_{\rho} \times_{M} J^{1} \Sigma_{\ell} \rightarrow \bigwedge^{4} T^{*} M, \quad \mathcal{L}_{\mathrm{EC}}\left(\theta, j^{1} \omega\right):=-\frac{1}{2 \kappa} \Omega_{a b} \wedge \Sigma^{a b},
$$

where $\left.\left.\kappa:=8 \pi G / c^{4}, \Sigma_{a b}:=e_{b}\right\lrcorner\left(e_{a}\right\lrcorner \Sigma\right)$ and $\Sigma$ is the standard volume form on $M$ locally given by $\operatorname{det}\|\theta\| \mathrm{d} x^{0} \wedge \cdots \wedge \mathrm{d} x^{3}$. Here $\|\theta\|$ stands for the matrix of the components of $\theta$ and we have set $e_{a}:=e_{a}{ }^{\mu} \partial_{\mu},\left\|e_{a}{ }^{\mu}\right\|$ denoting the inverse of $\|\theta\|$. The Dirac Lagrangian reads instead

$$
\mathcal{L}_{\mathrm{D}}: \Sigma_{\rho} \times_{M} \Sigma_{\ell} \times_{M} J^{1} \Sigma_{\hat{\gamma}} \rightarrow \bigwedge^{4} T^{*} M, \quad \mathcal{L}_{\mathrm{D}}\left(\theta, \omega, j^{1} \psi\right):=\left[\frac{\mathrm{i} \alpha}{2}\left(\bar{\psi} \gamma^{a} \nabla_{a} \psi-\nabla_{a} \bar{\psi} \gamma^{a} \psi\right)-m \bar{\psi} \psi\right] \Sigma,
$$

where $\alpha:=\hbar c$. According to the principle of minimal coupling, the total Lagrangian of the theory will be simply assumed to be $\mathcal{L}:=\mathcal{L}_{\mathrm{EC}}+\mathcal{L}_{\mathrm{D}}$. A vertical vector field on the configuration bundle will then read

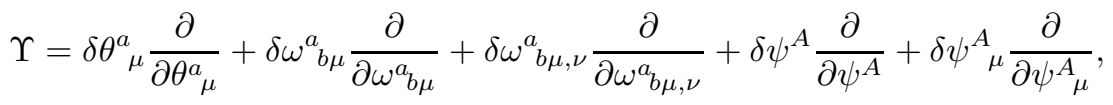

where $\left(\theta^{a}{ }_{\mu}\right),\left(\omega_{b \mu}^{a}, \omega_{b \mu, \nu}^{a}\right)$ and $\left(\psi^{A}, \psi_{\mu}^{A}\right)$ denote fibre coordinates on $\Sigma_{\rho}, J^{1} \Sigma_{\ell}$ and $J^{1} \Sigma_{\hat{\gamma}}$, respectively. If we set locally

$$
\begin{aligned}
\delta \theta^{a} & :=\delta \theta^{a}{ }_{\mu} \mathrm{d} x^{\mu}, & \delta \omega^{a}{ }_{b} & :=\delta \omega^{a}{ }_{b \mu} \mathrm{d} x^{\mu}, \\
\delta \psi & :=\delta \psi^{A} f_{A}, & \delta \bar{\psi} & :=\overline{\delta \psi},
\end{aligned}
$$

$\left(f_{A}\right)$ denoting a local fibre basis of $\Sigma_{\hat{\gamma}}$, then the first variation formula for $\mathcal{L}$ is

$$
\begin{aligned}
\delta \mathcal{L}=\left(-\frac{1}{\kappa} G_{b}^{a}+T_{b}^{a}\right) \Sigma_{a} & \wedge \delta \theta^{b}+\left(\frac{1}{2 \kappa} \mathcal{D} \Sigma_{a b}-S_{a b}{ }^{c} \Sigma_{c}\right) \wedge \delta \omega^{a b} \\
+ & \mathrm{d}_{\mathrm{H}}\left[-\frac{1}{2 \kappa} \Sigma_{a b} \wedge \delta \omega^{a b}-\frac{\mathrm{i} \alpha}{2}\left(\delta \bar{\psi} \gamma^{a} \psi-\bar{\psi} \gamma^{a} \delta \psi\right) \Sigma_{a}\right] \\
& +\alpha\left[\delta \bar{\psi} \mathbb{E}\left(\mathcal{L}_{\mathrm{D}}\right)+\overline{\mathbb{E}}\left(\mathcal{L}_{\mathrm{D}}\right) \delta \psi\right] \Sigma,
\end{aligned}
$$

where $G$ denotes the Einstein tensor associated with $\left.\Omega, \Sigma_{a}:=e_{a}\right\lrcorner \Sigma$ and we set

$$
\begin{array}{rlrl}
T_{b}^{a} & :=\Theta^{a}{ }_{b}-\frac{\alpha}{2}\left[\bar{\psi} \mathbb{E}^{\prime}\left(\mathcal{L}_{\mathrm{D}}\right)+\overline{\mathbb{E}}^{\prime}\left(\mathcal{L}_{\mathrm{D}}\right) \psi\right] \delta^{a}{ }_{b}, & \Theta^{a}{ }_{b} & :=\frac{\mathrm{i} \alpha}{2}\left(\bar{\psi} \gamma^{a} \nabla_{b} \psi-\nabla_{b} \bar{\psi} \gamma^{a} \psi\right), \\
\mathbb{E}^{\prime}\left(\mathcal{L}_{\mathrm{D}}\right) & :=\mathrm{i} \gamma^{a} \nabla_{a} \psi-m \psi, & \overline{\mathbb{E}}^{\prime}\left(\mathcal{L}_{\mathrm{D}}\right) & :=\overline{\mathbb{E}^{\prime}\left(\mathcal{L}_{\mathrm{D}}\right)} \equiv-\left(\mathrm{i} \nabla_{a} \bar{\psi} \gamma^{a}+m \bar{\psi}\right), \\
\mathbb{E}\left(\mathcal{L}_{\mathrm{D}}\right) & :=\mathbb{E}^{\prime}\left(\mathcal{L}_{\mathrm{D}}\right)-\frac{\mathrm{i}}{2} K_{b a}^{a} \gamma^{b} \psi, & \overline{\mathbb{E}}\left(\mathcal{L}_{\mathrm{D}}\right):=\overline{\mathbb{E}\left(\mathcal{L}_{\mathrm{D}}\right)} \equiv \overline{\mathbb{E}}^{\prime}\left(\mathcal{L}_{\mathrm{D}}\right)+\frac{\mathrm{i}}{2} K_{b a}^{a} \bar{\psi} \gamma^{b}, \\
S^{a b c}:=-\frac{\mathrm{i} \alpha}{8} \bar{\psi}\left(\gamma^{a b} \gamma^{c}+\gamma^{c} \gamma^{a b}\right) \psi \equiv-\frac{\mathrm{i} \alpha}{4} \bar{\psi} \gamma^{a b c} \psi \equiv S^{[a b c]},
\end{array}
$$

identity (1.2) having been used in the last but one equality. Thus, the Einstein-Cartan-Dirac equations are

$$
\begin{gathered}
G_{a b} \approx \kappa T_{a b}, \\
\mathcal{D} \Sigma_{a b} \approx 2 \kappa S_{a b}{ }^{c} \Sigma_{c}, \\
\mathrm{i} \gamma^{a} \nabla_{a} \psi-m \psi-\frac{\mathrm{i}}{2} K^{a}{ }_{b a} \gamma^{b} \psi \approx 0 .
\end{gathered}
$$


The first two equations are called the first and the second Einstein-Cartan (-Dirac) equation, respectively, whereas the last one is known as the (Cartan-) Dirac equation. $T$ is the energymomentum tensor of the theory, and $S$ the spin momentum tensor. Now, making use of (5.4), the second Einstein-Cartan equation can be put into the form

$$
\tau^{c} \wedge \Sigma_{a b c} \approx 2 \kappa S_{a b}^{c} \Sigma_{c}
$$

or equivalently

$$
\tau^{a b c} \approx 2 \kappa S^{a b c},
$$

which in turn implies that the torsion tensor is completely antisymmetric on shell. Therefore, so is the contortion tensor. Indeed, from (5.5) and (5.8)

$$
K^{a b c} \approx-\frac{1}{2} \tau^{a b c} \approx-\kappa S^{a b c}
$$

Hence, the Dirac equation reduces to $\mathbb{E}^{\prime}\left(\mathcal{L}_{\mathrm{D}}\right) \approx 0$, which implies $T_{a b} \approx \Theta_{a b}$. To sum up, the above system of equations is completely equivalent to the following

$$
\begin{gathered}
G_{a b} \approx \kappa \Theta_{a b}, \\
\tau^{a b c} \approx 2 \kappa S^{a b c}, \\
\mathrm{i} \gamma^{a} \nabla_{a} \psi-m \psi \approx 0 .
\end{gathered}
$$

Comparison between (3.2) and (3.6) tells us that we can read off the Noether current associated with $\mathcal{L}$ from its first variation $(5.7)$ :

$$
E(\mathcal{L}, \Xi)=-\xi\lrcorner \mathcal{L}-\frac{1}{2 \kappa} \Sigma_{a b} \wedge £_{\Xi} \omega^{a b}-\frac{\mathrm{i} \alpha}{2}\left(£_{\Xi} \bar{\psi} \gamma^{a} \psi-\bar{\psi} \gamma^{a} £_{\Xi} \psi\right) \Sigma_{a} .
$$

After some manipulation, which makes use (inter alia) of the fact that the (formal) Lie derivative (4.3b) of $\omega$ can be put into the form

$$
\left.£_{\Xi} \omega^{a}{ }_{b}=\xi\right\lrcorner \Omega^{a}{ }_{b}+\mathcal{D} \check{\Xi}_{b}^{a},
$$

$\left(\check{\Xi}^{a}{ }_{b}:=\Xi^{a}{ }_{b}+\omega^{a}{ }_{b \mu} \xi^{\mu}\right)$ being the components of the vertical part of $\Xi_{\tilde{\Lambda}}$ with respect to $\omega$, and that $\left.G_{b}^{a} \Sigma_{a} \equiv-1 / 2 \Omega^{a c} \wedge\left(e_{b}\right\lrcorner \Sigma_{a c}\right), E(\mathcal{L}, \Xi)$ can be recast as

$$
E(\mathcal{L}, \Xi)=\xi^{b}\left(-\frac{1}{\kappa} G_{b}^{a}+T_{b}^{a}\right) \Sigma_{a}+\check{\Xi}^{a b}\left(\frac{1}{2 \kappa} \mathcal{D} \Sigma_{a b}-S_{a b}{ }^{c} \Sigma_{c}\right)+\mathrm{d}_{\mathrm{H}}\left(-\frac{1}{2 \kappa} \check{\Xi}^{a b} \Sigma_{a b}\right),
$$

so that the superpotential associated with $\mathcal{L}$ turns out to be

$$
U(\mathcal{L}, \Xi):=-\frac{1}{2 \kappa} \check{\Xi}^{a b} \Sigma_{a b},
$$

a result which appeared in [22] for the first time. Therefore, the Dirac Lagrangian does not seem to contribute to the total superpotential. From this fact one might mistakenly conclude that the Dirac fields do not contribute to the total conserved quantities. This conclusion would be wrong because, although the Dirac Lagrangian does not contribute directly to the superpotential, in order to obtain the corresponding conserved quantities, one needs integrate the superpotential on a solution, which in turn depends on the Dirac Lagrangian via its energy-momentum tensor and the second Einstein-Cartan equation.

Note that in the case of the "Kosmann lift" [11] (see also [20]) we have

$$
\check{\Xi}^{a b}=\left(\check{\xi}_{\mathrm{K}}\right)^{a b} \equiv-\tilde{\nabla}^{[a} \xi^{b]},
$$

' $\tilde{\nabla}$ ' denoting the covariant derivative with respect to the transposed connection. Substituting (5.11) into (5.10) gives a half of the well-known "Komar potential" [29], in accordance with the result originally found by Kijowski [26] (see also [16]) in a purely natural context. This is also the lift implicitly used in $[12,21]$. 


\subsubsection{Natural approach}

Suppose for a moment that we deliberately neglected the gauge-natural nature of the EinsteinCartan-Dirac theory. This means that we shall temporarily regard the Einstein-Cartan Lagrangian as a purely natural Lagrangian, i.e. a first order Lagrangian on a (purely) natural bundle. In particular, the spin-connection $\omega$ will be replaced by a linear connection $\Gamma$, i.e. a principal connection on $L M$, the latter regarded as a natural bundle over $M$ (cf. Remark 2.2). As such, $\Gamma$ is a natural object, whose components $\left(\Gamma_{\nu \mu}^{\rho}\right)$ are related, because of (5.3), to the components $\left(\omega^{a}{ }_{b \mu}\right)$ of $\omega$ via the familiar formula ${ }^{5}$

$$
\omega_{b \mu}^{a}=\theta_{\rho}^{a}\left(\partial_{\mu} e_{b}{ }^{\rho}+\Gamma_{\nu \mu}^{\rho} e_{b}{ }^{\nu}\right),
$$

where antisymmetrization in $\{a, b\}$ is understood on the right-hand side of (5.12). Note that we cannot regard the Dirac Lagrangian itself as a natural Lagrangian because spinors cannot be suitably replaced by any (physically equivalent) natural objects: this is precisely why we went for a gauge-natural formulation in the first place, and why we expect to encounter some sort of restrictions now.

The local expression for the Lie derivative of $\Gamma$ reads

$$
£_{\xi} \Gamma^{\rho}{ }_{\nu \mu}:=£_{\Xi} \Gamma_{\nu \mu}^{\rho}=R^{\rho}{ }_{\nu \sigma \mu} \xi^{\sigma}+\nabla_{\mu} \tilde{\nabla}_{\nu} \xi^{\rho},
$$

a formula that has been known for a long time (cf., e.g., $[40,48])$ and can be evaluated directly from (2.4) or, equivalently, starting from (5.9) and then using (5.12) and (4.3a). Thus, the Noether current is now of the form

$$
E(\mathcal{L}, \Xi)=-\xi\lrcorner \mathcal{L}-\frac{1}{2 \kappa} \Sigma_{a b} \wedge £_{\xi} \Gamma^{a b}-\frac{\mathrm{i} \alpha}{2}\left(£_{\Xi} \bar{\psi} \gamma^{a} \psi-\bar{\psi} \gamma^{a} £_{\Xi} \psi\right) \Sigma_{a},
$$

where we set

$$
£_{\xi} \Gamma^{a b}:=\left(£_{\xi} \Gamma_{\nu \mu}^{\rho}\right) \theta_{\rho}^{a} \theta^{b \nu} \mathrm{d} x^{\mu} .
$$

The important point to note here is that, although $\left(\Gamma^{\rho}{ }_{\nu \mu}\right)$ may be regarded as the components of $\omega$ in a holonomic basis, $\left(£_{\xi} \Gamma_{\nu \mu}^{\rho}\right)$ are not, in general, the components of $\Xi_{\Xi} \omega$ in the corresponding basis. Accordingly, the second term on the right-hand side of identity (5.14) cannot be claimed to be the most general expression for $\left\langle f\left(\mathcal{L}_{\mathrm{EC}}\right), £_{\Xi} \omega\right\rangle$, but naturality must indeed be assumed. In fact, if we now proceeded in the same way as before, we would then find that consistency with the second Einstein-Cartan equation requires $\check{\Xi}^{a b}=-\tilde{\nabla}^{[a} \xi^{b]}$, i.e. precisely the Kosmann lift, and thus we would recover the purely natural result, as expected.

\subsection{Einstein-Dirac theory}

Our main reference for the Einstein-Dirac theory is [31]. The procedure for obtaining the conserved quantities is completely analogous to the Einstein-Cartan-Dirac case; therefore, we shall limit ourselves to present the results and briefly comment on them, pointing out the possible differences. In the sequel, the symbol ' $\left.\right|_{K=0}$ ' affixed to a quantity shall mean that the latter is formally identical with the quantity denoted by the same letter in $\S 5.2$, but with all (explicit or implicit) occurrences of $\omega$ replaced by ${ }^{\theta} \omega$.

Then, the Einstein-Hilbert Lagrangian is nothing but

$$
\mathcal{L}_{\mathrm{EH}}: J^{2} \Sigma_{\rho} \rightarrow \bigwedge^{4} T^{*} M, \quad \mathcal{L}_{\mathrm{EH}}\left(j^{2} \theta\right):=\left.\mathcal{L}_{\mathrm{EC}}\right|_{K=0},
$$

whereas the Dirac Lagrangian is regarded here as the base-preserving morphism

$$
{ }^{\theta} \mathcal{L}_{\mathrm{D}}: J^{1} \Sigma_{\rho} \times{ }_{M} J^{1} \Sigma_{\hat{\gamma}} \rightarrow \bigwedge^{4} T^{*} M, \quad{ }^{\theta} \mathcal{L}_{\mathrm{D}}\left(j^{1} \theta, j^{1} \psi\right):=\left.\mathcal{L}_{\mathrm{D}}\right|_{K=0} .
$$

\footnotetext{
${ }^{5}$ Note that formula (5.12) is formally identical — not surprisingly — with the usual expression for the components of the pull-back of $\Gamma$ on $M$ in an (anholonomic) orthonormal basis. Note also that, for $\Gamma_{\nu \mu}^{\rho}$, we use a different subscript ordering from [27].
} 
Again, the total Lagrangian of the theory will simply be ${ }^{\theta} \mathcal{L}:=\mathcal{L}_{\mathrm{EH}}+{ }^{\theta} \mathcal{L}_{\mathrm{D}}$, its variation reading

$$
\begin{aligned}
\delta^{\theta} \mathcal{L}=\left(-\frac{1}{\kappa}{ }^{\theta} G_{b}^{a}+{ }^{\theta} T^{a}{ }_{b}\right) \Sigma_{a} & \wedge \delta \theta^{b} \\
+\mathrm{d}_{\mathrm{H}}\left[-\frac{1}{2 \kappa} \Sigma_{a b}\right. & \left.\wedge \delta^{\theta} \omega^{a b}+\frac{1}{2} S^{a b}{ }_{c} \Sigma_{a b} \wedge \delta \theta^{c}-\frac{\mathrm{i} \alpha}{2}\left(\delta \bar{\psi} \gamma^{a} \psi-\bar{\psi} \gamma^{a} \delta \psi\right) \Sigma_{a}\right] \\
& +\alpha\left[\delta \bar{\psi} \mathbb{E}\left({ }^{\theta} \mathcal{L}_{\mathrm{D}}\right)+\overline{\mathbb{E}}\left({ }^{\theta} \mathcal{L}_{\mathrm{D}}\right) \delta \psi\right] \Sigma
\end{aligned}
$$

where

$$
\begin{aligned}
{ }^{\theta} T^{a}{ }_{b} & :=\left.T^{a}{ }_{b}\right|_{K=0}+{ }^{\theta} \nabla_{c} S^{a}{ }_{b}{ }^{c} \\
& \equiv{ }^{\theta} \Theta^{a}{ }_{b}+\bar{b}^{a}{ }_{b} \mathbb{E}\left({ }^{\theta} \mathcal{L}_{\mathrm{D}}\right)+\overline{\mathbb{E}}\left({ }^{\theta} \mathcal{L}_{\mathrm{D}}\right) b^{a}{ }_{b}, \\
{ }^{\theta} \Theta^{a}{ }_{b} & :=\frac{1}{2}\left(\Theta^{a}{ }_{b}+\Theta_{b}{ }^{a}\right)_{K=0} \equiv{ }^{\theta} \Theta_{b}{ }^{a}, \\
b^{a}{ }_{b} & :=\frac{\alpha}{4}\left(\gamma^{a}{ }_{b}-2 \delta^{a}{ }_{b}\right) \psi, \quad \bar{b}^{a}{ }_{b}:=\overline{b^{a}{ }_{b}} \equiv-\frac{\alpha}{4} \bar{\psi}\left(\gamma^{a}{ }_{b}+2 \delta^{a}{ }_{b}\right), \\
\mathbb{E}\left({ }^{\theta} \mathcal{L}_{\mathrm{D}}\right):= & \left.\mathbb{E}^{\prime}\left(\mathcal{L}_{\mathrm{D}}\right)\right|_{K=0}, \quad \overline{\mathbb{E}}\left({ }^{\theta} \mathcal{L}_{\mathrm{D}}\right):=\left.\overline{\mathbb{E}\left({ }^{\theta} \mathcal{L}_{\mathrm{D}}\right)} \equiv \overline{\mathbb{E}}^{\prime}\left(\mathcal{L}_{\mathrm{D}}\right)\right|_{K=0} .
\end{aligned}
$$

Thus, the Einstein-Dirac equations are

$$
\begin{gathered}
{ }^{\theta} G_{a b} \approx \kappa^{\theta} \Theta_{a b}, \\
\mathrm{i} \gamma^{a}{ }^{\theta} \nabla_{a} \psi-m \psi \approx 0 .
\end{gathered}
$$

Note that, although the invariance of the Dirac Lagrangian with respect to Lorentz transformations requires ${ }^{\theta} T_{a b}$ to be symmetric on shell $[47,6]$, the manipulation required for going from (5.15) to (5.16) is highly non-trivial: the interested reader is referred to [31] for an elegant proof.

Following the same procedure as before, we find that the Noether current associated with ${ }^{\theta} \mathcal{L}$ is

$$
\begin{aligned}
E\left({ }^{\theta} \mathcal{L}, \Xi\right) & =-\xi\lrcorner{ }^{\theta} \mathcal{L}-\frac{1}{2 \kappa} \Sigma_{a b} \wedge £_{\Xi}{ }^{\theta} \omega^{a b}+\frac{1}{2} S^{a b}{ }_{c} \Sigma_{a b} \wedge £_{\Xi} \theta^{c}-\frac{\mathrm{i} \alpha}{2}\left(£_{\Xi} \bar{\psi} \gamma^{a} \psi-\bar{\psi} \gamma^{a} £_{\Xi} \psi\right) \Sigma_{a} \\
& =\xi^{b}\left(-\frac{1}{\kappa} G^{\theta} G_{b}^{a}+{ }^{\theta} T^{a}{ }_{b}\right) \Sigma_{a}+\mathrm{d}_{\mathrm{H}}\left(-\frac{1}{2 \kappa} \check{\Xi}^{a b} \Sigma_{a b}+\frac{1}{2} \xi^{c} S^{a b}{ }_{c} \Sigma_{a b}\right),
\end{aligned}
$$

so that the superpotential associated with ${ }^{\theta} \mathcal{L}$ is recognized to be

$$
U\left({ }^{\theta} \mathcal{L}, \Xi\right):=-\frac{1}{2 \kappa} \check{\Xi}^{a b} \Sigma_{a b}+\frac{1}{2} \xi^{c} S^{a b}{ }_{c} \Sigma_{a b},
$$

and we note that, unlike the Einstein-Cartan-Dirac case, the Dirac Lagrangian enters the superpotential directly, but recall that we have no second Einstein-Cartan equation here. Note also that the "vertical contribution" (i.e. all terms in $\check{\Xi}^{a b}$ ) coming from the Dirac Lagrangian consistently vanishes off shell. For the same reason, no inconsistency of the type of $\S 5.2 .1$ can arise here. This fact, though, by no means disproves the gauge-naturality of the theory, which is well-motivated on both physical and mathematical grounds.

\subsection{The indeterminacy}

Both (5.10) and (5.17) reveal that, in this gauge-natural formulation of gravity coupled with Dirac fields, the superpotential is essentially indeterminate because no condition can be imposed a priori on the vertical part of $\Xi$. Therefore, we can state our main result as follows.

Theorem 5.1. Any conserved charge associated with the gravitational field is intrinsically indeterminate.

Note that, because of (3.12), this indeterminacy does not depend on the particular Lagrangians chosen: for this reason and the functorial nature of this indeterminacy we have called it "intrinsic". This important result can be regarded either as a limit for the theory or as an additional flexibility (cf. [22]). In any case, it cannot be overlooked. 
From a physical point of view, it might be disturbing to think, that, when the spinorial contribution is removed, the (gravitational part of the) theory should automatically revert to its purely natural counterpart, thereby reproducing the well-known (non-indeterminate ${ }^{6}$ ) results. This could mean either that some justification has to be found to impose the Kosmann lift by hand or, conversely, as we believe, that a gauge-natural formulation is the appropriate one for gravity for the very reason that it is the most general one ${ }^{7}$, irrespectively of the nature of the theory it is possibly coupled with.

\section{Acknowledgements}

The author would like to express his deep gratitude to Dr. Marco Godina and Prof. James A. Vickers for their useful remarks and encouragement. Also, this paper is, in many respects, the climax of a long-term research project carried out over several years by the mathematical physics group of the Department of Mathematics of the University of Turin, Italy, whose indirect contribution to this work is hereby gratefully acknowledged.

\section{References}

[1] M. F. Atiyah. Geometry of Yang-Mills fields. Lezioni Fermiane. Scuola Normale Superiore, Pisa, 1979.

[2] G. Barnich and F. Brandt. Covariant theory of asymptotic symmetries, conservation laws and central charges. Nuclear Phys. B, 633(1-2):3-82, 2002.

[3] H. A. Buchdahl. On the compatibility of relativistic wave-equations in Riemann-Cartan spaces. J. Math. Phys., 30(3):700-5, 1989.

[4] H. A. Buchdahl. On gauge-invariant and phase-invariant spinor analysis. II. J. Math. Phys., 33(1):647, 1992.

[5] P. Budinich and A. Trautman. The spinorial chessboard. Trieste Notes in Physics. Springer-Verlag, Berlin, 1988.

[6] Y. Choquet-Bruhat. Spin 1/2 fields in arbitrary dimensions and the Einstein-Cartan theory. In W. Rindler and A. Trautman, editors, Gravitation and geometry. A volume in honour of Ivor Robinson, number 4 in Monogr. Textbooks Phys. Sci., pages 83-106. Bibliopolis, Napoli, 1987.

[7] D. J. Eck. Gauge-natural bundles and generalized gauge theories. Mem. Amer. Math. Soc., 33(247), 1981.

[8] D. J. Eck. Product preserving functors on smooth manifolds. J. Pure Appl. Algebra, 42(2):133-40, 1986.

[9] D. B. A. Epstein and W. P. Thurston. Transformation groups and natural bundles. Proc. London Math. Soc. (3), 38(2):219-36, 1979.

[10] L. Fatibene. Formalismo gauge-naturale per le teorie di campo classiche. Tesi di Dottorato, Università degli Studi di Torino, 1999.

[11] L. Fatibene, M. Ferraris, M. Francaviglia, and M. Godina. A geometric definition of Lie derivative for spinor fields. In J. Janyška, I. Kolář, and J. Slovák, editors, Proc. 6th International Conference on Differential Geometry and its Applications (Brno, 1995), pages 549-58, Brno, 1996. Masaryk University.

[12] L. Fatibene, M. Ferraris, M. Francaviglia, and M. Godina. Gauge formalism for general relativity and fermionic matter. Gen. Rel. Grav., 30(9):1371-89, 1998.

[13] L. Fatibene and M. Francaviglia. Deformations of spin structures and gravity. Acta Phys. Polon. B, 29(4):915-28, 1998.

\footnotetext{
${ }^{6}$ But not always satisfactory: see $[21], \S 4$.

${ }^{7}$ Note that, even if we were to couple Einstein (-Cartan) gravity with " $U_{4}$-spinors" $[3,4,23], \mathfrak{s o}(1,3)$ is in some sense "maximal" since the superpotential of the theory must be a 2 -form.
} 
Einstein-Dirac theory on gauge-natural bundles

[14] L. Fatibene and M. Francaviglia. Natural and gauge-natural field theories. In Geometry Seminars, 2000 (Bologna, 1999/2000), pages 123-50. Univ. Stud. Bologna, Bologna, 2001.

[15] L. Fatibene, M. Francaviglia, and M. Palese. Conservation laws and variational sequences in gaugenatural theories. Math. Proc. Cambridge Philos. Soc., 130(3):555-69, 2001.

[16] M. Ferraris, M. Francaviglia, and M. Mottini. Conserved quantities of the gravitational field in tetrad notation. Rend. Mat. Appl. (7), 14(3):457-81, 1994.

[17] M. Ferraris, M. Francaviglia, and O. Robutti. Energy and superpotentials in gravitational field theories. In M. Modugno, editor, Atti del $6^{\circ}$ Convegno Nazionale di Relatività Generale e Fisica della Gravitazione, pages 137-50, Bologna, 1986. Pitagora Editrice.

[18] M. Ferraris, M. Francaviglia, and O. Robutti. On the notion of energy and the existence of superpotentials in gravitational theories. In Y. Choquet-Bruhat, B. Coll, R. Kramer, and A. Lichnerowicz, editors, Géométrie et physique, Proc. Journées Relativistes (Marseille, 1985), pages 112-25. Hermann, Paris, 1987.

[19] G. Giachetta, L. Mangiarotti, and G. Sardanashvily. New Lagrangian and Hamiltonian methods in field theory. World Scientific, Singapore, 1997.

[20] M. Godina and P. Matteucci. Reductive G-structures and Lie derivatives. J. Geom. Phys., 47(1):6686, 2003. Erratum: Ibid., 48(1):133-4, 2003. E-print: arXiv:math.DG/0201235.

[21] M. Godina, P. Matteucci, L. Fatibene, and M. Francaviglia. Two-spinor formulation of first order gravity coupled to Dirac fields. Gen. Rel. Grav., 32(1):145-59, 2000. E-print: arXiv:gr-qc/9903023.

[22] M. Godina, P. Matteucci, and J. A. Vickers. Metric-affine gravity and the Nester-Witten 2-form. J. Geom. Phys., 39(4):265-75, 2001. E-print: arXiv:gr-qc/0006032.

[23] P. Godlewski. Bitetrad as geometrodynamical variable for GL(2, C)-invariant models of mutually interacting gravitational and bispinor fields. Rep. Math. Phys., 49(1):39-62, 2002.

[24] J. Janyška and I. Kolář. Lie derivatives on vector bundles. In O. Kowalski, editor, Proceedings of the Conference on Differential Geometry and its Applications (Nové Město na Moravě, 1980), pages 111-6, Prague, 1982. Univ. Karlova.

[25] G. Kainz and P. W. Michor. Natural transformations in differential geometry. Czechoslovak Math. J., 37(112)(4):584-607, 1987.

[26] J. Kijowski. On a new variational principle in general relativity and the energy of the gravitational field. Gen. Rel. Grav., 9(10):857-77, 1978.

[27] S. Kobayashi and K. Nomizu. Foundations of differential geometry, volume 1. Wiley, New York, 1963.

[28] I. Kolár̆, P. W. Michor, and J. Slovák. Natural operations in differential geometry. Springer-Verlag, Berlin, 1993.

[29] A. Komar. Covariant conservation laws in general relativity. Phys. Rev. (2), 113:934-6, 1959.

[30] D. Krupka and J. Musilová. Trivial Lagrangians in field theory. Differential Geom. Appl., 9(3):293305, 1998. Erratum: Ibid., 10(3):303, 1999.

[31] A. Lichnerowicz. Champ de Dirac, champ du neutrino et transformations C, P, T sur un espace-temps courbe. Ann. Inst. H. Poincaré Sect. A (N.S.), 1(3):233-90, 1964.

[32] O. O. Luciano. Categories of multiplicative functors and Weil's infinitely near points. Nagoya Math. J., 109:69-89, 1988.

[33] A. Nijenhuis. Natural bundles and their general properties. Geometric objects revisited. In Differential geometry (in honor of Kentaro Yano), pages 317-34. Kinokuniya, Tokyo, 1972.

[34] E. Noether. Invariante Variationsprobleme. Nachr. v. d. Ges. d. Wiss. zu Göttingen, pages 235-57, 1918.

[35] R. S. Palais and C. L. Terng. Natural bundles have finite order. Topology, 16(3):271-7, 1977.

[36] R. J. Plymen and B. W. Westbury. Complex conformal rescaling and spin-structure. Bull. London Math. Soc., 19(4):363-70, 1987.

[37] O. Robutti. Tensori energetici e superpotenziali nelle teorie geometriche di campo. Tesi di Laurea, Università degli Studi di Torino, 1984. 
[38] S. E. Salvioli. On the theory of geometric objects. J. Diff. Geom., 7:257-78, 1972.

[39] D. J. Saunders. The geometry of jet bundles. Number 142 in London Math. Soc. Lecture Notes. Cambridge University Press, Cambridge, 1989.

[40] J. A. Schouten. Ricci-calculus. An introduction to tensor analysis and its geometrical applications. Number 10 in Grundlehren der Mathematischen Wissenschaften. Springer-Verlag, Berlin, 2nd edition, 1954.

[41] J. J. Sławianowski. U(2,2)-invariant spinorial geometrodynamics. Rep. Math. Phys., 38(3):375-97, 1996.

[42] S. T. Swift. Spinors, embeddings and gravity. PhD thesis, University of Southampton, 1988.

[43] C. L. Terng. Natural vector bundles and natural differential operators. Amer. J. Math., 100(4):775828, 1978.

[44] A. Trautman. Invariance of Lagrangian systems. In L. O'Raifeartaigh, editor, General relativity. Papers in honour of J. L. Synge, pages 85-99. Clarendon Press, Oxford, 1972.

[45] A. Trautman. Fiber bundles, gauge fields, and gravitation. In A. Held, editor, General relativity and gravitation. One hundred years after the birth of Albert Einstein, volume 1, pages 287-308. Plenum Press, New York, 1980.

[46] B. M. van den Heuvel. Energy-momentum conservation in gauge theories. J. Math. Phys., 35(4):166887,1994

[47] S. Weinberg. Gravitation and cosmology: principles and applications of the general theory of relativity. Wiley, New York, 1972.

[48] K. Yano. The theory of Lie derivatives and its applications. North-Holland, Amsterdam, 1957. 